\title{
Small RNAs just got bigger: Piwi-interacting RNAs (piRNAs) in mammalian testes
}

\author{
V. Narry Kim ${ }^{1}$ \\ School of Biological Sciences and Institute of Molecular Biology and Genetics, Seoul National University, \\ Seoul, 151-742, Korea
}

Small RNAs constitute a large family of regulatory molecules with diverse functions in eukaryotes. Hallmarks of small RNAs are their dependence on double-stranded RNAs (dsRNA)-specific RNase III-type enzymes for biogenesis and their association with Argonaute family proteins for the silencing process. At least two classes of small RNAs have previously been described: microRNAs (miRNAs) derived from hairpin-shaped precursors and small interfering RNAs (siRNAs) generated from long dsRNAs. Recent articles reported a novel class of small RNAs that are expressed specifically and abundantly in the spermatogenic cells of mice. These RNAs are bigger (26-31 nucleotides [nt]) than most previously described small RNAs (21-23 nt) and are associated with Piwi-subclade members of the Argonaute protein family. Although the biogenesis and function of these RNAs are yet to be determined, these findings may add new dimensions in small RNA biology and germline cell biology.

\section{Different classes of small RNAs}

Eukaryotes produce various types of small RNAs of 1930 nucleotides (nt) in length that function in diverse pathways (Bartel 2004; Kim 2005; Brodersen and Voinnet 2006; Vaucheret 2006). Small RNAs act as guides to direct mRNA degradation, translational repression, heterochromatin formation, and DNA elimination. Because the active forms of small RNAs are often indistinguishable biochemically or functionally in some species, they are conventionally grouped based on their origins into two classes: microRNAs (miRNAs) and small interfering RNAs (siRNAs) (Fig. 1). miRNAs are generated from the dsRNA region of the hairpin-shaped precursors, whereas siRNAs are derived from long double-stranded RNAs (dsRNAs). Both miRNAs and siRNAs bind to mRNA and induce mRNA cleavage, translational repression, and cleavage-independent mRNA decay.

[Keywords: piRNA; miRNA; siRNA; Piwi; Argonaute; testis] ${ }^{1}$ Correspondence.

E-MAIL narrykim@snu.ac.kr; FAX 82-2-887-0244.

Article is online at http://www.genesdev.org/cgi/doi/10.1101/gad.1456106.
Several groups of natural siRNAs (Nat-siRNAs) have been described in various eukaryotic species. Nat-siRNAs in plants and endogenous siRNAs (endo-siRNAs) in nematodes are generated from dsRNAs derived from endogenous sense and antisense transcripts (Borsani et al. 2005; Lee et al. 2006). Trans-acting siRNAs (tasiRNAs) in plants are processed from dsRNAs that are synthesized by an RNA-dependent RNA polymerase using endogenous transcripts as the templates (Peragine et al. 2004). Endogenous tasiRNAs direct cleavage of endogenous cognate mRNAs in trans (the target genes are different from the gene that the siRNA originates). Tiny noncoding RNAs (tncRNAs) are tasiRNA-like RNAs found in nematode worms (Ambros et al. 2003; Lee et al. 2006). Small scan RNAs (scnRNAs) are thought to scan for regions in DNA for genome rearrangement in Tetrahymena thermophila (Mochizuki et al. 2002; Taverna et al. 2002; Liu et al. 2004). The Argonaute family protein, Twilp, mediates the process and a Dicer homolog is required for the accumulation of scnRNAs (Mochizuki et al. 2002). Repeat-associated siRNAs (rasiRNAs), on the other hand, are roughly defined as small RNAs that match repetitive sequence elements in sense and antisense orientations (Aravin et al. 2001, 2003, 2004; Djikeng et al. 2001; Hall et al. 2002; Hamilton et al. 2002; Llave et al. 2002; Mette et al. 2002; Pal-Bhadra et al. 2002; Reinhart and Bartel 2002; Volpe et al. 2002). RasiRNAs are presumably derived from long dsRNAs, but their biogenesis mechanism remains unclear. RasiRNAs may function in the establishment of heterochromatin in repetitive elements.

In mammals, only the miRNA class has been identified, and there have been no descriptions of any other class of endogenous small RNAs. This review will summarize recent findings of a previously unknown class of small RNAs specifically expressed in mammalian germline cells (Aravin et al. 2006; Girard et al. 2006; Grivna et al. 2006; Watanabe et al. 2006).

Piwi subfamily proteins and spermatogenesis

Argonaute family proteins are the prime components of small RNA complexes that are highly conserved among 


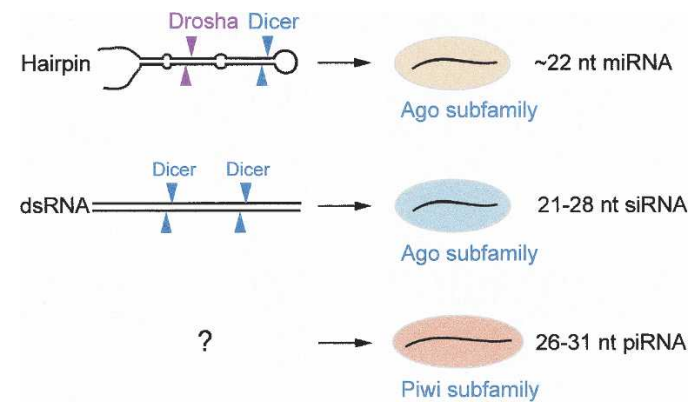

Figure 1. Classification of small RNAs. Definition and classification of small RNAs conventionally relies on their biogenesis mechanism. Two relatively well-defined classes of small RNAs include microRNAs (miRNAs) and small interfering RNAs (siRNAs). The biogenesis mechanism for piRNAs is currently unknown.

eukaryotes (Hammond et al. 2001; Carmell et al. 2002; Mourelatos et al. 2002; Sontheimer and Carthew 2004). Argonaute proteins are defined by the PAZ ( $\underline{\text { Piwi }}$ Argonaut and $\underline{Z} w i l l e)$ domain and the PIWI domain. The results of several structural and biochemical studies indicated that Argonaute proteins are capable of interacting directly with small RNAs through their PAZ domains (to the $3^{\prime}$ end of small RNA) and through the PIWI and the middle domains (to the $5^{\prime}$ end of small RNA) (Song et al. 2003, 2004; Yan et al. 2003; Lingel et al. 2004; Ma et al. 2004; Rivas et al. 2005). A small RNA guides the Argonaute protein to its target molecule, which leads to gene silencing.

The Argonaute family can be divided into Ago and Piwi subclades based on amino acid sequence similarities. In general, the Ago members are expressed ubiquitously and are associated with miRNAs and siRNAs. In contrast, the Piwi members are more restricted in germline cells and stem cells. Until recently, the RNAs associated with Piwi members remained unidentified.

The expression patterns and knockout phenotypes of mammalian Piwi members indicated that they play important roles during spermatogenesis (Fig. 2; KuramochiMiyagawa et al. 2001; Deng and Lin 2002). Mammalian spermatogenesis can be divided into three phases. The first phase is mitotic self-renewing of germline stem cells (spermatogonia), producing primary spermatocytes. The second phase is the meiosis of primary spermatocytes, which generates haploid round spermatids. The prophase of the first meiotic division progresses in the following order: leptotene, zygotene, pachytene, diplotene, and diakinesis. During the third phase (called spermiogenesis), the round spermatids undergo reorganization and morphological changes into spermatozoa.

There are four Ago members (AGO1 4) and three piwi members (MIWI, MILI/PIWIL2, and MIWI2/PIWIL4) in mice. The expression kinetics of MILI and MIWI are different (Fig. 2; Kuramochi-Miyagawa et al. 2001; Deng and Lin 2002). MILI is expressed until the pachytene spermatocyte stage, whereas MIWI is detected later from the mid-pachytene stage to early round spermatids.
Their expression overlaps in the mid-pachytene stage. Spermatogenetic arrest points are also different in MILInull and MIWI-null mice (Fig. 2). MILI-null mice are arrested at the early pachytene spermatocyte phase, whereas MIWI-null mice are arrested at the round spermatid stage.

It is unclear whether Drosophila piwi is the ortholog of mammalian Piwi proteins. Fly PIWI is expressed in the nuclei of germline cells as well as of the supporting somatic cells, whereas MIWI and MILI are found in the cytoplasm of germ cells (Cox et al. 1998). In addition, fly piwi mutant shows defects not only in spermatogenesis but also in the maintenance of germline stem cells (Cox et al. 1998). Drosophila aub, another member of the piwi subfamily, may be a closer homolog to Miwi and Mili because AUB is found in the cytoplasm of spermatogonia and spermatocytes. Loss of aub function leads to abnormal development of spermatocytes and round spermatids (Schmidt et al. 1999).

\section{Discovery of piRNAs}

Piwi-interacting RNAs (piRNAs) were recently isolated from mouse testes independently by four groups (Aravin et al. 2006; Girard et al. 2006; Grivna et al. 2006; Watanabe et al. 2006). In three laboratories, the initial recognition of $\sim 30$-nt piRNAs was made by simply looking at the total testis RNA on ethidium bromide (or SYBR green)-stained gel because piRNAs are much more abundant in testes than other small RNA species (Aravin et al. 2006; Girard et al. 2006; Grivna et al. 2006; Watanabe et al. 2006). In fact, it was later estimated that approximately one million piRNA molecules exist per spermatocyte or round spermatid (Aravin et al. 2006). Subsequent cloning led to the discovery of numerous small RNAs that range from 26 to $\sim 31 \mathrm{nt}$.

With an aim of identifying MIWI-associated small RNA, Hannon and colleagues (Girard et al. 2006) first visualized testes RNA by pCp labeling at the $3^{\prime}$ end (Grivna et al. 2006). Intrigued by the abundant RNAs of unexpected size (29-30 nt), they went on to sequence the RNAs. Apart from conventional cDNA cloning method, Hannon and colleagues also employed highly

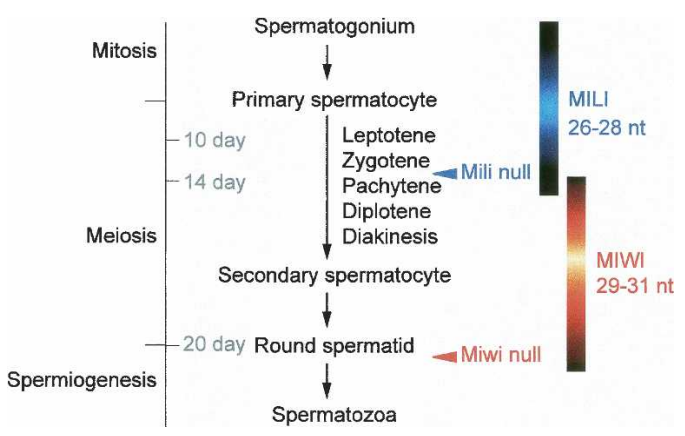

Figure 2. Expression of piRNAs and Piwi subfamily members during mouse spermatogenesis. Mammalian spermatogenesis occurs within the seminiferous epithelium inside the seminiferous tubule and can be divided into three phases. 
parallel pyrosequencing technology to obtain $>50,000$ RNA sequences for which they coined the name "piwi-interacting RNAs" (piRNAs). Despite intensive sequencing, many sequences were found only once, indicating that the piRNA population is extremely diverse.

Similarly, Tuschl and colleagues (Aravin et al. 2006) investigated MILI-associated RNAs. By immunoprecipitation from testis lysate using anti-MILI antibody, they found that 26- to 28-nt RNAs are associated with MILI and that there are additional 29- to 31-nt RNA species that do not interact with MILI. Subsequent cloning resulted in $>15,000$ sequences.

Imai and colleagues (Watanabe et al. 2006) cloned small RNAs not only from mouse testes but also from mouse oocytes. Cloning of testis small RNA yielded 381 clones of $\sim 27$-nt RNAs. From oocytes, they isolated seven miRNAs and 40 potential rasiRNAs that are $\sim 22 \mathrm{nt}$ in length. This suggests that piRNAs may have a specific role in male germline, although it is possible that oocyte-specific RNAs were underrepresented in the sample due to the limited number of oocytes in mouse ovaries.

Lin and colleagues (Grivna et al. 2006) noticed that the 30-nt RNA band was not detectable in their Miwi-null mice (Girard et al. 2006). The investigators then cloned 29- to 34-nt RNA isolated from total testicular RNA and discovered 40 different RNAs ranging from 25 to $35 \mathrm{nt}$ (mainly 29-31 nt). Among these, two overlapped with the Imai set (Watanabe et al. 2006). These RNAs were precipitated specifically with anti-MIWI antibody, indicating that MIWI is associated with this class of RNA and is required for their accumulation.

Size distribution of cloned RNAs indicated that there are two distinct populations of piRNAs (Aravin et al. 2006; Girard et al. 2006; Grivna et al. 2006; Watanabe et al. 2006). piRNAs of the first group are 29-31 nt in length and associated with MIWI protein. Consistent with their dependence on Miwi, their expression coincides with that of MIWI, which is from the mid-pachytene to the early round spermatid stage (Fig. 2). This group of piRNAs can be detected from day 15 mice but does not reach the maximal level until day 18. The second group includes slightly shorter piRNAs (26-28 nt in length) that are preferentially associated with MILI (Fig. 2; Aravin et al. 2006). Detected abundantly by day 14, MILI-associated RNAs are expressed as early as from spermatogonia and begin to diminish after the round spermatid stage. In adult mouse testes, the first group (29- to 31-nt piRNA) is more abundant than the second group (26-28 nt). These two groups may be generated from the same loci via differential processing because the probes for some piRNAs can detect both 30-nt band and 26-nt band (Aravin et al. 2006; Watanabe et al. 2006). Determination of the termini by rapid amplification of cDNA ends (RACE) also revealed that the $5^{\prime}$ ends of piRNA clones were invariable while the $3^{\prime}$ ends were shorter by $2 \mathrm{nt}$ in MILI-associated RNA clones (Aravin et al. 2006). Whether these two groups of RNAs play distinct roles remains to be determined.

\section{Genomic distribution and characteristics of piRNAs}

Cloned piRNAs show rather uneven distribution among chromosomes (Aravin et al. 2006; Girard et al. 2006; Grivna et al. 2006; Watanabe et al. 2006). They are enriched in chromosomes $17,5,4$, and 2 but seem to be largely excluded from sex chromosomes. They are slightly enriched in intergenic regions that are poor in genes or repeats. Although $\sim 17 \%$ of piRNAs were mapped to repeat sequences (mostly corresponding to retrotransposons), this proportion is lower than expected by random sampling of 30-nt sequences from the genome ( 40\%). Imai and colleagues (Watanabe et al. 2006) classified these repeat-matching RNAs in testes into rasiRNAs. However, judging from their size distribution, at least some of these repeat-matching RNAs may need to be recategorized into the piRNA class.

The vast majority of piRNAs $196 \%$ in Hannon set; $89 \%$ in Imai and Lin set; $81 \%$ in Tuschl set) are clustered in relatively short genomic loci, which range from $<1 \mathrm{~kb}$ to $>100 \mathrm{~kb}$ and contain 10-4500 small RNAs (Fig. 3). Importantly, almost all piRNAs in a given cluster are derived from the same orientation. This extreme strand bias indicates that piRNAs may be processed from a long primary transcript. In some clusters, one strand is changed abruptly to another strand (Fig. 3), suggesting that these bidirectional clusters may be transcribed divergently from a central promoter.

Because small RNAs are usually defined and classified based on their dsRNA origin, one may argue that these RNAs may be the degradation products from large transcripts or belong to an unrelated RNA family. However, the following characteristics strongly advocate that piRNAs are authentic members of the small RNA family. Firstly, their lengths distribute in a significantly narrow range. Northern blotting also showed discrete bands representing either 26- to 28-nt or 29- to 31-nt RNAs. Secondly, as mentioned earlier, they are physically associated with Argonaute family proteins, which are the key factors in RNA silencing pathways. Thirdly, their $5^{\prime}$ ends have a strong preference for uracil $(\sim 86 \%)$ that is characteristic of small RNAs. For instance, $\sim 76 \%$ of miRNAs have $U$ at the $5^{\prime}$-most position. The reason for this sequence bias remains unclear, but it has been proposed that RNA processing factors such as Dicer and Drosha cleave preferentially at the 5' side of U.

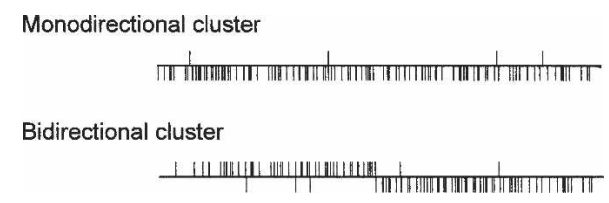

Figure 3. Strand bias of piRNAs. The first and second tracks indicate piRNAs mapping to the negative and positive strands, respectively. Monodirectional clusters encode piRNAs mainly on only one strand, whereas bidirectional clusters encode piRNAs on two strands that are segregated into two separate parts. 


\section{Unanswered questions and future directions}

It remains elusive how these 26- to 35-nt RNAs are generated. So far, there is no evidence that these RNAs are generated from dsRNA precursor. The strong strand bias of clustered piRNAs suggests that they may be generated from the single-stranded precursor. Supporting this notion, both EST analysis and RT-PCR experiments revealed putative primary transcripts but failed to detect the antisense transcripts (Watanabe et al. 2006). Unlike miRNA precursors, the regions covering piRNA clones do not fold into stem-loop structures. Therefore, the biogenesis pathway of piRNAs appears to be distinct from that of miRNAs and siRNAs. Several possible mechanisms have been proposed for piRNA biogenesis. First, long-range dsRNA structures may be formed in primary transcripts, which may explain the strand bias of piRNAs (Aravin et al. 2006). Second, the antisense transcript may be expressed at such a low level that they escape detection (Watanabe et al. 2006). Third, primary transcripts may be digested by a ssRNA-directed unidentified enzyme. Fourth, Imai and colleagues (Watanabe et al. 2006) proposed that piRNAs may be derived from RNA-DNA duplexes that are produced by the reverse transcriptase of retrotransposons.

Although it was previously shown that human Piwi proteins can interact with Dicer (Sasaki et al. 2003), it has yet to be determined whether RNase III-type enzymes such as Dicer and Drosha are indeed involved in piRNA biogenesis. Conditional knockout of Dicer and Drosha will help answer this question. Biochemical analysis of Piwi complex will also be required to identify the biogenesis factors for piRNA.

Perhaps the most crucial question is as to the function of piRNAs. Because null mice of Miwi and Mili show clear defects in spermatogenic cells, piRNAs may be involved in spermatogenesis possibly by regulating meiosis and/or suppressing retrotransposons. One possibility is that piRNAs act like miRNAs by guiding the Piwi complex to their target mRNAs whose activity is required for spermatogenesis regulation. However, most piRNAs are not complementary to mRNA, suggesting that they are unlikely to function as post-translational regulators of protein synthesis. In fact, it is conceivable that the target molecules may not be RNA molecules. Interestingly, individual piRNA sequences are not conserved, whereas piRNA loci are conserved in the syntenic regions of other mammalian species (Girard et al. 2006). This led to the suggestion that piRNA generation process itself from these loci may be significant for the function (Girard et al. 2006). Identification of the target molecules of the piRNA-Piwi complex will be a crucial step toward understanding the functions of piRNAs. Genetic analyses on these loci will be necessary to elucidate the function of piRNAs. It will also be imperative to reveal the other protein components of the piRNA-Piwi complex. Biochemical and cell biological analysis of Piwi proteins will provide insights into these enigmatic and intriguing RNA species.

\section{Acknowledgments}

I thank Inha Heo and Dong-Hyeok Kim for their assistance with manuscript preparation. This work was supported by the Basic Research Program (R02-2004-000-10173-0), the National Research Laboratory Program (M1050000010905J000010910), the Korea Research Foundation Grant (KRF-2005-005-J16001), and the BK21 program.

\section{Note added in proof}

Recent work by Kingston and colleagues (Lau et al. 2006) also described piRNAs from rat testes. Rat piRNAs are associated with the piRNA complex (piRC), which contains Riwi, the rat homolog to Piwi, as well as rRecQ1, a homolog to QDE-3 from Neurospora that has been implicated in RNA silencing pathways.

\section{References}

Ambros, V., Lee, R.C., Lavanway, A., Williams, P.T., and Jewell, D. 2003. MicroRNAs and other tiny endogenous RNAs in $C$. elegans. Curr. Biol. 13: 807-818.

Aravin, A.A., Naumova, N.M., Tulin, A.V., Vagin, V.V., Rozovsky, Y.M., and Gvozdev, V.A. 2001. Double-stranded RNAmediated silencing of genomic tandem repeats and transposable elements in the D. melanogaster germline. Curr. Biol. 11: 1017-1027.

Aravin, A.A., Lagos-Quintana, M., Yalcin, A., Zavolan, M., Marks, D., Snyder, B., Gaasterland, T., Meyer, J., and Tuschl, T. 2003. The small RNA profile during Drosophila melanogaster development. Dev. Cell 5: 337-350.

Aravin, A.A., Klenov, M.S., Vagin, V.V., Bantignies, F., Cavalli, G., and Gvozdev, V.A. 2004. Dissection of a natural RNA silencing process in the Drosophila melanogaster germ line. Mol. Cell. Biol. 24: 6742-6750.

Aravin, A., Gaidatzis, D., Pfeffer, S., Lagos-Quintana, M., Landgraf, P., Iovino, N., Morris, P., Brownstein, M.J., KuramochiMiyagawa, S., Nakano, T., et al. 2006. A novel class of small RNAs bind to MILI protein in mouse testis. Nature (in press).

Bartel, D.P. 2004. MicroRNAs: Genomics, biogenesis, mechanism, and function. Cell 116: 281-297.

Borsani, O., Zhu, J., Verslues, P.E., Sunkar, R., and Zhu, J.K. 2005. Endogenous siRNAs derived from a pair of natural cis-antisense transcripts regulate salt tolerance in Arabidopsis. Cell 123: 1279-1291.

Brodersen, P. and Voinnet, O. 2006. The diversity of RNA silencing pathways in plants. Trends Genet. 22: 268-280.

Carmell, M.A., Xuan, Z., Zhang, M.Q., and Hannon, G.J. 2002. The Argonaute family: Tentacles that reach into RNAi, developmental control, stem cell maintenance, and tumorigenesis. Genes \& Dev. 16: 2733-2742.

Cox, D.N., Chao, A., Baker, J., Chang, L., Qiao, D., and Lin, H. 1998. A novel class of evolutionarily conserved genes defined by piwi are essential for stem cell self-renewal. Genes \& Dev. 12: 3715-3727.

Deng, W. and Lin, H. 2002. miwi, a murine homolog of piwi, encodes a cytoplasmic protein essential for spermatogenesis. Dev. Cell 2: 819-830.

Diikeng, A., Shi, H., Tschudi, C., and Ullu, E. 2001. RNA interference in Trypanosoma brucei: Cloning of small interfering RNAs provides evidence for retroposon-derived 24-26nucleotide RNAs. RNA 7: 1522-1530.

Girard, A., Sachidanandam, R., Hannon, G.J., and Carmell, M.A. 
2006. A germline-specific class of small RNAs binds mammalian Piwi proteins. Nature (in press).

Grivna, S.T., Beyret, E., Wang, Z., and Lin, H. 2006. A novel class of small RNAs in mouse spermatogenic cells. Genes \& Dev. 20: 1709-1714.

Hall, I.M., Shankaranarayana, G.D., Noma, K., Ayoub, N., Cohen, A., and Grewal, S.I. 2002. Establishment and maintenance of a heterochromatin domain. Science 297: 2232-2237.

Hamilton, A., Voinnet, O., Chappell, L., and Baulcombe, D. 2002. Two classes of short interfering RNA in RNA silencing. EMBO J. 21: 4671-4679.

Hammond, S.M., Boettcher, S., Caudy, A.A., Kobayashi, R., and Hannon, G.J. 2001. Argonaute2, a link between genetic and biochemical analyses of RNAi. Science 293: 1146-1150.

Kim, V.N. 2005. Small RNAs: Classification, biogenesis, and function. Mol. Cells 19: 1-15.

Kuramochi-Miyagawa, S., Kimura, T., Yomogida, K., Kuroiwa, A., Tadokoro, Y., Fujita, Y., Sato, M., Matsuda, Y., and Nakano, T. 2001. Two mouse piwi-related genes: miwi and mili. Mech. Dev. 108: 121-133.

Lau, N.C., Seto, A.G., Kim, J., Kuramochi-Miyagawa, S., Nakano, T., Bartel, D.P., and Kingston, R.E. 2006. Characterization of the piRNA complex from rat testes. Science (in press). [Epub ahead of print June 15, 2006.]

Lee, R.C., Hammell, C.M., and Ambros, V. 2006. Interacting endogenous and exogenous RNAi pathways in Caenorhabditis elegans. RNA 12: 589-597.

Lingel, A., Simon, B., Izaurralde, E., and Sattler, M. 2004. Nucleic acid 3'-end recognition by the Argonaute2 PAZ domain. Nat. Struct. Mol. Biol. 11: 576-577.

Liu, Y., Mochizuki, K., and Gorovsky, M.A. 2004. Histone H3 lysine 9 methylation is required for DNA elimination in developing macronuclei in Tetrahymena. Proc. Natl. Acad. Sci. 101: 1679-1684.

Llave, C., Kasschau, K.D., Rector, M.A., and Carrington, J.C. 2002. Endogenous and silencing-associated small RNAs in plants. Plant Cell 14: 1605-1619.

Ma, J.B., Ye, K., and Patel, D.J. 2004. Structural basis for overhang-specific small interfering RNA recognition by the PAZ domain. Nature 429: 318-322.

Meister, G., Landthaler, M., Patkaniowska, A., Dorsett, Y., Teng, G., and Tuschl, T. 2004. Human Argonaute2 mediates RNA cleavage targeted by miRNAs and siRNAs. Mol. Cell 15: 185-197.

Mette, M.F., van der Winden, J., Matzke, M., and Matzke, A.J. 2002. Short RNAs can identify new candidate transposable element families in Arabidopsis. Plant Physiol. 130: 6-9.

Mochizuki, K., Fine, N.A., Fujisawa, T., and Gorovsky, M.A. 2002. Analysis of a piwi-related gene implicates small RNAs in genome rearrangement in tetrahymena. Cell 110: 689699.

Mourelatos, Z., Dostie, J., Paushkin, S., Sharma, A., Charroux, B., Abel, L., Rappsilber, J., Mann, M., and Dreyfuss, G. 2002. miRNPs: A novel class of ribonucleoproteins containing numerous microRNAs. Genes \& Dev. 16: 720-728.

Pal-Bhadra, M., Bhadra, U., and Birchler, J.A. 2002. RNAi related mechanisms affect both transcriptional and posttranscriptional transgene silencing in Drosophila. Mol. Cell 9: 315327.

Peragine, A., Yoshikawa, M., Wu, G., Albrecht, H.L., and Poethig, R.S. 2004. SGS3 and SGS2/SDE1/RDR6 are required for juvenile development and the production of trans-acting siRNAs in Arabidopsis. Genes \& Dev. 18: 2368-2379.

Reinhart, B.J. and Bartel, D.P. 2002. Small RNAs correspond to centromere heterochromatic repeats. Science 297: 1831.

Rivas, F.V., Tolia, N.H., Song, J.J., Aragon, J.P., Liu, J., Hannon,
G.J., and Joshua-Tor, L. 2005. Purified Argonaute2 and an siRNA form recombinant human RISC. Nat. Struct. Mol. Biol. 12: 340-349.

Sasaki, T., Shiohama, A., Minoshima, S., and Shimizu, N. 2003. Identification of eight members of the Argonaute family in the human genome small star, filled. Genomics 82: 323-330.

Schmidt, E.E., Hanson, E.S., and Capecchi, M.R. 1999. Sequence-independent assembly of spermatid mRNAs into messenger ribonucleoprotein particles. Mol. Cell. Biol. 19: 3904-3915.

Song, J.J., Liu, J., Tolia, N.H., Schneiderman, J., Smith, S.K., Martienssen, R.A., Hannon, G.J., and Joshua-Tor, L. 2003. The crystal structure of the Argonaute2 PAZ domain reveals an RNA binding motif in RNAi effector complexes. Nat. Struct. Biol. 10: 1026-1032.

Song, J.J., Smith, S.K., Hannon, G.J., and Joshua-Tor, L. 2004. Crystal structure of Argonaute and its implications for RISC slicer activity. Science 305: 1434-1437.

Sontheimer, E.J. and Carthew, R.W. 2004. Molecular biology. Argonaute journeys into the heart of RISC. Science 305: 1409-1410.

Taverna, S.D., Coyne, R.S., and Allis, C.D. 2002. Methylation of histone h3 at lysine 9 targets programmed DNA elimination in tetrahymena. Cell 110: 701-711.

Vaucheret, H. 2006. Post-transcriptional small RNA pathways in plants: Mechanisms and regulations. Genes \& Dev. 20: 759-771.

Volpe, T.A., Kidner, C., Hall, I.M., Teng, G., Grewal, S.I., and Martienssen, R.A. 2002. Regulation of heterochromatic silencing and histone H3 lysine-9 methylation by RNAi. Science 297: 1833-1837.

Watanabe, T., Takeda, A., Tsukiyama, T., Mise, K., Okuno, T., Sasaki, H., Minami, N., and Imai, H. 2006. Identification and characterization of two novel classes of small RNAs in the mouse germline: Retrotransposon-derived siRNAs in oocytes and germline small RNAs in testes. Genes \& Dev. 20: 17321743.

Yan, K.S., Yan, S., Farooq, A., Han, A., Zeng, L., and Zhou, M.M. 2003. Structure and conserved RNA binding of the PAZ domain. Nature 426: 468-474. 


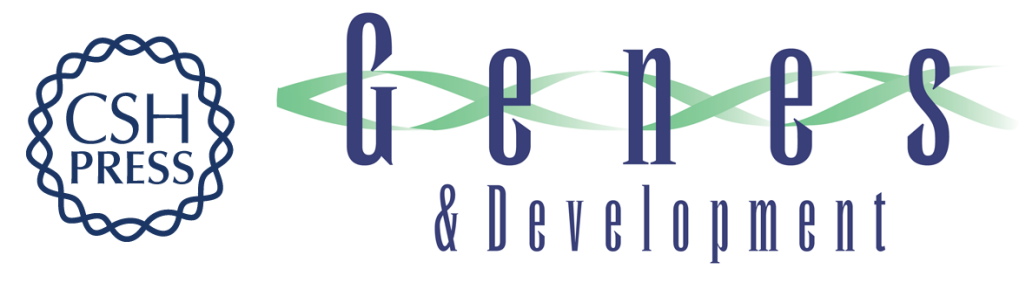

\title{
Small RNAs just got bigger: Piwi-interacting RNAs (piRNAs) in mammalian testes
}

\author{
V. Narry Kim \\ Genes Dev. 2006, 20: \\ Access the most recent version at doi:10.1101/gad.1456106
}
Related Content A novel class of small RNAs in mouse spermatogenic cells
Shane T. Grivna, Ergin Beyret, Zhong Wang, et al.
Genes Dev. UNKNOWN , 2006 20: 1709-1714 Identification and characterization of two novel classes of small RNAs in the mouse germline: retrotransposon-derived siRNAs in oocytes and germline small RNAs in testes
Toshiaki Watanabe, Atsushi Takeda, Tomoyuki Tsukiyama, et al.
Genes Dev. UNKNOWN , 2006 20: 1732-1743 Pimet, the Drosophila homolog of
HEN1, mediates 22-0-methylation of Piwi- interacting RNAs at their 32 ends
Kuniaki Saito, Yuriko Sakaguchi, Takeo Suzuki, et al.
Genes Dev. July , 2007 21: 1603-1608 Specific association of Piwi with rasiRNAs
derived from retrotransposon and heterochromatic regions in the Drosophila genome
Kuniaki Saito, Kazumichi M. Nishida, Tomoko Mori, et al.
Genes Dev. August , 2006 20: 2214-2222

References This article cites 40 articles, 21 of which can be accessed free at:

http://genesdev.cshlp.org/content/20/15/1993.full.html\#ref-list-1

Articles cited in:

http://genesdev.cshlp.org/content/20/15/1993.full.html\#related-urls

\section{License}

Email Alerting

Receive free email alerts when new articles cite this article - sign up in the box at the top Service

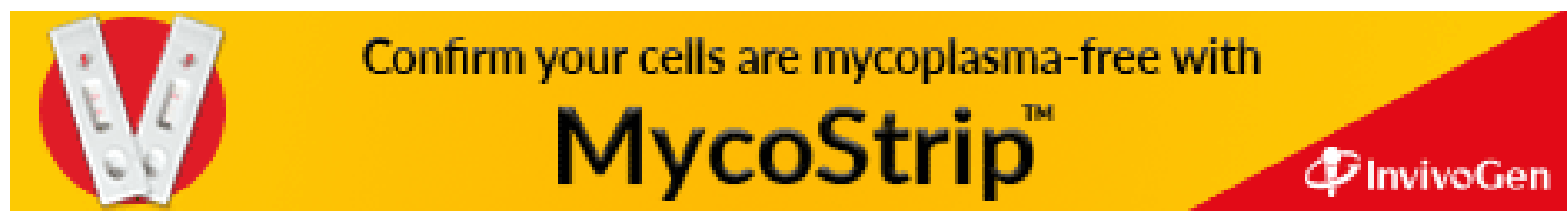

